\title{
ANALYSIS OF HYDROGEN DEPLETION USING A SCALED PASSIVE AUTOCATALYTIC RECOMBINER *
}

\author{
Thomas K. Blanchat \\ Reactor Safety Experiments \\ Sandia National Laboratories \\ Albuquerque, NM 87185-1139 \\ Asimios Malliakos \\ U.S. Nuclear Regulatory Commission \\ Washington, DC 20555-0001
}

\begin{abstract}
Hydrogen depletion tests of a scaled passive autocatalytic recombiner (PAR) were performed in the Surtsey test vessel at Sandia National Laboratories (SNL). The experiments were used to determine the hydrogen depletion rate of a PAR in the presence of steam and also to evaluate the effect of scale (number of cartridges) on the PAR performance at both low and high hydrogen concentrations.
\end{abstract}

\section{INTRODUCTION}

PARs are being considered by the nuclear power industry as a combustible gas control system in operating plants and advanced light water reactor (ALWR) containments for design basis events. PARs do not require a source of power; instead, they use a catalyst to recombine hydrogen and oxygen gases into water vapor upon contact with the catalyst. At low hydrogen concentrations, energy from the recombination of hydrogen with oxygen is released at a relatively slow (but continuous) rate into the containment. The heat produced creates strong buoyancy effects which increases the influx of the surrounding gases to the recombiner. These natural convective flow currents promote mixing of combustible gases in the containment. The recombination rate of the PAR system needs to be great enough to keep the concentration of hydrogen below acceptable levels.

There are several catalytic recombiner concepts under development worldwide. SNL is evaluating a PAR which has been developed by the NIS Company, in Hanau, Germany. Detailed tests and analyses were made in cooperation with the Battelle Institute, Frankfurt, and the Technical University, Munich. Its development has been sponsored by the German utility, RWE Energie. The NIS/RWE PAR device contains flat rectangular cartridges filled with porous spherical ceramic pellets, which are coated with palladium. The large surface area of the palladium layer of the pellets acts on diffused gas molecules to recombine hydrogen with oxygen.

\footnotetext{
* This work was supported by the U.S. Nuclear Regulatory Commission and was performed at Sandia National Laboratories. Sandia is a multiprogram laboratory operated by Sandia Corporation, a Lockheed Martin Company, for the U.S. Department of Energy under Contract DE-AC04-94AL85000.
} 


\section{DISCLAIMER}

This report was prepared as an account of work sponsored by an agency of the United States Government. Neither the United States Government nor any agency thereof, nor any of their employees, make any warranty, express or implied, or assumes any legal liability or responsibility for the accuracy, completeness, or usefulness of any information, apparatus, product, or process disclosed, or represents that its use would not infringe privately owned rights. Reference herein to any specific commercial product, process, or service by trade name, trademark, manufacturer, or otherwise does not necessarily constitute or imply its endorsement, recommendation, or favoring by the United States Government or any agency thereof. The views and opinions of authors expressed herein do not necessarily state or reflect those of the United States Government or any agency thereof. 


\section{DISCLAIMER}

\section{Portions of this document may be illegible in electronic image products. Images are produced from the best available original document.}


Between the cartridges, the PAR device has open flow channels to allow heavier particles or aerosols in the atmosphere to flow through with little plugging of the pellet surface.

SNL, under the sponsorship and direction of the USNRC, has conducted an experimental program to evaluate the performance of PARs. A PAR was tested at the Surtsey experimental test facility at SNL. The following describes the configuration of the PAR, the test facility, the instrumentation, the control and data acquisition system, the test conditions, and the test results and analyses.

\section{PAR AND TEST FACILITY DESCRIPTION}

The PAR test module was a scaled version of the prototype PAR that was developed and fabricated by NIS INGENIEURGESELLSCHAFT MBH (Hanau, Germany). ${ }^{1}$ The prototype PAR contained two rows of standard catalytic cartridges (44 cartridges per row) and had dimensions of $1 \mathrm{~m}$ by $1 \mathrm{~m}$. The PAR test module (also manufactured by NIS) contained only one row of standard catalytic cartridges and could be assembled as either a $1 / 2$ scale (44 cartridges), $1 / 4$ scale ( 22 cartridges), or $1 / 8$ scale ( 11 cartridges) PAR by removing cartridges and using smaller (length) front and back panels. Note that the $1 / 2$ scale PAR test module configuration has dimensions of $\sim 0.5 \mathrm{~m}$ by $\sim 1.0 \mathrm{~m}$.

Figures 1 and 2 show that the PAR test module housing holds the catalyst cartridges in a vertical plane and guides the air flow. A vertical flow channel of about $1-\mathrm{cm}$ spacing is formed between the cartridges. These flow channels (along with the PAR body or housing) define the flow area for convection of the heat generated by the heat of reaction. The PAR exit has a chimney with a free cross-sectional area equal to the cross-sectional area through the cartridges.

The catalyst material is inserted into rectangular cartridges $(0.45$ meter length, $0.01 \mathrm{~m}$ wide, $0.20 \mathrm{~m}$ tall). The cartridges are filled with the catalyst pellets. The steel sides of the cartridges are perforated with many slotted-like openings that allow hydrogen to enter into the cartridge. The catalyst is a palladium-coated $(0.5 \mathrm{w} / \mathrm{o})$ aluminum oxide pellet with a diameter of about $4-6 \mathrm{~mm}$ and a bulk density of $\sim 0.5 \mathrm{~g} / \mathrm{cc}$. The porous oxide pellet provides a large inside surface area $\left(-100 \mathrm{~m}^{2} / \mathrm{g}\right)$ of palladium that allows a high conversion. A hydrophobic coating is placed on each pellet to minimize startup delays due to water on the catalyst surface, either from steam condensation or from containment sprays.

Figure 3 shows the location of the PAR test module in the Surtsey vessel. The PAR was located at the vessel centerline, $-1 \mathrm{~m}$ above the midline elevation in the Surtsey vessel. Horizontal and vertical I-beams exist in the lower half of the Surtsey vessel but there are no Ibeams located directly below the PAR. The Surtsey vessel is an ASME-approved steel pressure vessel with a current working internal volume of $99 \mathrm{~m}^{3}$. It has a cylindrical shape with removable, dished heads attached to both ends, and is $3.6 \mathrm{~m}$ in diameter by $10.3 \mathrm{~m}$ high. The Surtsey vessel has a maximum allowable working pressure of $1 \mathrm{MPa}$ at $533 \mathrm{~K}$. A total of twenty $30.5-\mathrm{cm}(12$-inch) and $61-\mathrm{cm}(24-\mathrm{inch})$ instrument penetration ports exist at six different levels around the perimeter of the vessel. The vessel walls and heads are 5/8-in thick and covered with at least 4 inches of fiberglass insulation, or equivalent material. 


\section{INSTRUMENTATION, CONTROL, AND DATA ACQUISITION}

The most significant variables measured in the PAR experiments were: (1) the pressure and temperature in the Surtsey vessel, (2) the gas constituents and steam concentrations, (3) the PAR pellet and channel gap temperatures, (4) the flow velocity through the PAR, and (5) the amounts of hydrogen and oxygen injected into the vessel. A personal computer (PC)-based data acquisition system was designed to control and monitor the course of the test in real-time. The PC-based data acquisition (DAQ) system gave instantaneous readouts of the temperatures of the cartridge pellets and corresponding cartridge air gaps, Surtsey vessel pressure, temperatures, and gas concentrations, and valve positions for steam, hydrogen, and oxygen additions. In addition, the DAQ system controlled the hydrogen target concentration and gas addition interval. This allowed changing the test conditions (during the course of a test) based on real-time test results.

Four pressure transducers were used to measure the pressure in the Surtsey vessel. The gas temperature in the Surtsey vessel was measured with twenty thermocouples installed in two rakes. The two thermocouple rakes were installed vertically in the vessel; one rake at the vessel centerline (array A) and one rake (array B) located about $0.32 \mathrm{~m}$ from the vessel wall. Ten equally-spaced type- $\mathrm{K}$ thermocouples (1.0 m spacing) were located on each rake. Six type-K thermocouples were installed in the Surtsey vessel steel walls. In addition, thermocouples measured the injected oxygen, hydrogen, and steam temperatures, both at the respective manifolds and also at each steam/gas diffuser. In order to minimize steam condensation, steam was mixed with the oxygen and/or hydrogen during each gas injection.

A real-time gas mass spectroscopy (GMS) system was used to determine the concentrations of nitrogen, oxygen, and hydrogen in the vessel at four sample points. The four sample points were at the PAR inlet, the PAR outlet, high in the vessel near the dome, and low in the vessel near the floor. To ensure representative samples and to minimize the delay time due to purging sample lines, each line was purged for $\sim 1$ minute prior to sampling. This necessitated a continuous purge of gas out of the vessel. The sample lines and purge rates were sized to allow no more than a $1.0 \%$ loss (by volume) of gas out of the vessel over the course of a 12 hour test. Since the PAR inlet was the sample point of greatest interest, this point was selected for every other sample (i.e., PAR inlet, PAR outlet, PAR inlet, dome, PAR inlet, Surtsey floor, PAR inlet, PAR outlet, ...).

Ten to twenty (depending on test conditions) pre-evacuated $500-\mathrm{cm}^{3}$ gas grab sample bottles were used to collect samples from the vessel. Most of the gas grab samples were taken at the PAR inlet; however, any of the four gas sample points could have been selected. These gas grab samples were used as an independent verification of the gas composition.

A high resolution 1/2-in charge-coupled device (CCD) color camera was mounted on a level 5 port, and viewed the PAR through a tempered glass window. In addition to the digital camera, an infrared (IR) camera also viewed the PAR through a different level 5 port. The camera view could see the PAR exit (top of the chimney) and provide visual evidence in the event of a deflagration event. Other instrumentation included a hygrometer to measure relative 
humidity and pitot-tube differential pressure transducers and a hot-wire anemometer to measure the velocity of the gas at the PAR inlet and outlet.

Twelve thermocouples monitored the catalyst temperature at three cartridge locations; PAR middle (and a PAR middle backup), PAR edge, and PAR corner. Three vertical positions for temperature measurement were monitored at each location (2-cm from the bottom, middle, and $2-\mathrm{cm}$ from the top). These thermocouples were inserted into the cartridges and surrounded by the catalyst pellets. Twelve thermocouples monitored the temperature of the gas in the gap between the cartridges and were located opposite of the catalyst thermocouples. Four thermocouples monitored the PAR inlet temperature. Two thermocouples were located at the centerline middle and two were located at the centerline edge (within 2-cm of the PAR bottom). Four thermocouples monitored the PAR outlet temperature. Two thermocouples were located at the centerline middle and two were located at the centerline edge (within 2-cm of the chimney exit).

The hydrogen and oxygen gas was supplied to the vessel from separate manifolds. Standard 44 liters compressed gas cylinders were installed on the manifolds. In the tests that involve a prototypic air/steam atmosphere, the cold gas entering the vessel was mixed with an appropriate amount of steam (capable of heating the cold gas to near the desired test temperature) using a diffuser/mixer pipe that was located near the floor of the vessel. This was necessary to prevent condensation of the steam. Mass flow controllers were used to provide precise metering of the hydrogen and oxygen into the vessel. Two mixing fans were installed in the vessel. They were located on opposite sides of the PAR at the openings of the I-beam lattice; one pointed upward and one pointed downward. The fans were usually operated when hydrogen was injected and prior to taking gas grab samples.

\section{GAS COMPOSITION MEASUREMENTS AND ANALYSES}

The GMS system cannot measure steam concentrations; a dry sample must be presented. In order to achieve this, a condenser and condensate trap (and heated gas inlet lines) were installed on each gas measurement line. This yielded dry-basis gas concentrations; however, to determine wet-basis gas concentrations, the steam fraction must be known. A hygrometer was used to determine the relative humidity $(\mathrm{RH})$. The steam concentration was then calculated from the ratio of saturation pressure to total pressure times the RH fraction. The saturation pressure of steam was determined from saturated steam tables using the vessel average gas temperature. The thermocouples on array B were used to determine the vessel average temperature.

The nitrogen-ratio method was used to determine wet-basis gas concentrations as a second independent method. ${ }^{2}$ The nitrogen-ratio method does not require an estimate of the posttest noncondensible fraction. It does, however, require the pretest noncondensible fraction. For the various posttest times, the number of moles of nitrogen is assumed to be unchanged, and the number of moles of another gas is simply the number of moles of nitrogen gas times the ratio of the other gas specie mole fraction to the nitrogen mole fraction. The nitrogen-ratio method calculates the total number of noncondensible moles. Total moles in the vessel is calculated using ideal gas law relationships. Therefore, the number of steam moles is simply the difference 
between the total vessel moles and the total noncondensible moles. The steam fraction is found from the ratio of steam moles to total vessel moles.

\section{TEST MATRIX}

Six depletion rate tests using a scaled PAR were conducted. PAR performance at low hydrogen concentrations was determined at $1 / 2$ scale, at $1 / 4$ scale, and at $1 / 8$ scale. NIS states that the hydrophobic coating is probably destroyed when the PAR catalyst exceeds temperatures of about $473 \mathrm{~K}$. The PAR catalyst would reach these temperatures at about $2 \% \mathrm{H}_{2}$ in cold dry air and about $1 \% \mathrm{H}_{2}$ in the hot air/steam environment. These tests were performed at hydrogen concentrations that would not destroy the hydrophobic coating.

Three repeat tests (at $1 / 2$ scale, at $1 / 4$ scale, and at $1 / 8$ scale) were performed at relatively high hydrogen concentrations which yielded the scaled counterpart performance data and completed the scaled depletion rate test series.

\section{EXPERIMENTAL RESULTS}

The scaled depletion rate tests started with a mixture of $0.107 \mathrm{MPa}$ of air and $0.107 \mathrm{MPa}$ of steam, for a total pressure of about $0.21 \mathrm{MPa}$. To achieve these conditions, the vessel was sealed with about $0.083 \mathrm{MPa}$ of cold air inside (one Albuquerque atmosphere at about $293 \mathrm{~K}$ ). The Surtsey vessel was then heated internally with steam to obtain a gas temperature of about $375 \mathrm{~K}$. A portable steam boiler provided a low-pressure, saturated steam heat source $(227 \mathrm{~kg} / \mathrm{hr})$ into Surtsey. Initially, the steam that entered Surtsey condensed and yielded latent heat to the vessel walls and atmosphere. A steam trap installed in the Surtsey floor removed the condensate without removing gases. As the vessel heated, the vessel pressurized with steam. The vessel bulk gas and wall heat up was accomplished in about 12 hours. At a temperature of $375 \mathrm{~K}$, the air and steam pressures become equal. Note that the air pressure increased from $0.083 \mathrm{MPa}$ to $0.107 \mathrm{MPa}$ because of the increase in air temperature from $293 \mathrm{~K}$ to $375 \mathrm{~K}$.

Steam fractions, as determined from the ratio of saturation steam pressure to total pressure in the Surtsey vessel, and also from the nitrogen ratio method, were very similar during the course of the majority of the tests. A few deviations were seen in some tests and the steam fraction determined by the method of pressure ratio is suspect; these deviations were attributed to nonsaturated conditions in the Surtsey vessel that occurred after hydrogen burns or after operating the PAR at high hydrogen concentrations for long periods of time.

Two observations can be made regarding most of the PAR tests. The PAR started within 10 minutes in hot, steamy atmospheres when exposed to hydrogen concentrations in the range of 1 to 6 mole \%. The second observation was that at steady-state operation the PAR appeared to generate a convective flow loop in the Surtsey vessel from the PAR outlet to the dome, down the Surtsey wall (until reaching the height of the PAR inlet), and then returning to the PAR inlet; as indicated by both the hydrogen concentration and the vessel gas temperature measurements. Since the convection flow pattern did not extend to the lower half of Surtsey vessel, the vessel was not completely well-mixed by the PAR during steady-state operation. The hydrogen 
concentration from the sample point located near the floor always showed a higher concentration when measurements were taken after the last addition, as compared to the other sample points. This indicated that the depletion below the PAR near the floor was lower than that in the upper half of the Surtsey vessel. Also, the convective loop appeared to be driven further downward into the lower half of the Surtsey vessel in those tests with the higher hydrogen concentrations.

A test with the PAR configured at $1 / 8$ scale was used to measure the hydrogen depletion rate at low hydrogen concentration. The Surtsey vessel was sealed and pressurized with steam to about $0.21 \mathrm{MPa}$. The initial gas temperature was about $374 \mathrm{~K}$. The PAR started recombining after the first hydrogen addition to about 1.0 mole \%. There was about a ten minute delay (from the time of first hydrogen injection) in PAR startup. The startup is shown by the increase in the vessel gas temperature (Figures 4 and 5), the increase in the catalyst temperature (Figure 6), and the increase in the gas temperature at the PAR outlet (Figure 7). Figure 5 also shows the times when the mixing fans were operating while Figure 7 shows the hydrogen additions using the mass flow controller. Figure 8 gives the wet-basis gas concentrations. The steam concentration ranged from about 50 mole $\%$ at the beginning of the test to about $40 \mathrm{~mole} \%$ at the end. The oxygen concentration remained relatively constant at about 10 mole \%. Figure 9 focuses on the hydrogen concentrations at the vessel floor, PAR inlet, PAR outlet, and vessel dome locations. This figure also shows the integrated hydrogen addition. The hydrogen concentration from the sample point located near the floor showed smaller decreases as time progressed after the last addition, compared to the other sample points. This indicated that a reduced depletion occurred in the lower half of the Surtsey vessel, below the PAR elevation.

\section{PAR PERFORMANCE ANALYSES}

Hydrogen depletion rates are used to measure the performance of a PAR. The hydrogen depletion rate is usually determined as a function of the hydrogen concentration in the vessel. Depletion rate analyses can also be used to show the effect of various factors, such as PAR location, oxygen concentration, catalyst poison, etc., on PAR performance.

The following procedure was used to determine the depletion rate. First, the timedependent amount of hydrogen in the Surtsey vessel (in moles) was determined by multiplying the average hydrogen concentration by the total number of moles in the Surtsey vessel. The average hydrogen concentration was assumed to be that measured by the gas mass spectrometer at the PAR inlet sample point. The total number of moles in the vessel was calculated using the average gas temperature (determined from the array B thermocouples) and the ideal gas law.

As shown earlier in the results section, the hydrogen concentrations measured at the four sample locations diverged from some initially equal value over the course of a test. This was because the mixing fans were not turned on while depletion rate data were taken and the PAR flow was not sufficient to maintain a mixed condition in the vessel. The methodology used to determine the depletion rate assumed that the vessel was well-mixed; this introduced some error since the average hydrogen concentration was not actually measured and cannot be calculated since the local steam concentrations were not known. The measured depletion rates may slightly overpredict hydrogen consumption at the stated hydrogen level since the hydrogen concentration 
at the PAR inlet sample point, just before the fans were turned on, was lower than the average value. The depletion rate was then determined by calculating the difference in hydrogen moles at each successive time interval, using the data from the steady-state depletion interval, after the hydrogen additions were stopped. The calculated depletion rate was then plotted against the measured hydrogen concentration.

PAR performance and the effects of scale were determined with tests at both low and high hydrogen concentrations. Note that the initial conditions for all tests started with a vessel pressure of about $0.2 \mathrm{MPa}$, with approximately 50/50 mixtures of air and steam.

Figure 10 shows the PAR performance with low hydrogen concentrations $(<0.7$ mole $\%)$ at $1 / 2$ scale, $1 / 4$ scale, and $1 / 8$ scale. A regression fit of the number of hydrogen moles in the vessel during the steady-state depletion was used to calculate the fitted depletion rates (along with the $95 \%$ confidence intervals). Note that the $1 / 2$ scale depletion rate is $\sim 4$ times the $1 / 8$ scale depletion rate. Figure 11 shows the PAR performance with high hydrogen concentrations (1-6 mole \%) and at 1/2 scale, 1/4 scale, and 1/8 scale. Simple scaling does not appear to apply to depletion rates at high hydrogen concentrations.

A better comparison of the scaled depletion rate data can be made by normalizing the data. Depletion rate models predict that depletion rate is directly proportional to scale and depletion rate is independent of volume. ${ }^{3,4}$ Therefore, a simple scale factor can be used to normalize the data. These models assume that the vessel is well-mixed. However, if the PAR only consumes hydrogen in a small portion of the total vessel volume, the depletion rate calculation can overpredict consumption if the vessel is not well-mixed at all times. The depletion rate measurements then become scale-dependent since tests with larger scale and/or higher concentrations appear to deplete larger pockets of hydrogen within the total vessel volume.

Figures 12 and 13 show the scaled depletion rate data normalized to full-scale by applying the scale factor ( $\mathrm{x} 2$ for $1 / 2$ scale, $\mathrm{x} 4$ for $1 / 4$ scale, and $\mathrm{x} 8$ for $1 / 8$ scale). Figure 12 shows that the depletion rates for these tests of low hydrogen concentrations are indeed directly proportional to scale. However, Figure 13 shows that tests with high hydrogen concentrations yield lower depletion rates for larger scale, probably because larger volumes are being depleted.

Figures 12 and 13 also give comparisons with published depletion rate data at pressures of $0.2 \mathrm{MPa}$ and $0.1 \mathrm{MPa}$. Data at 0.1 MPa is only presented to highlight the physics; depletion of hydrogen by the PAR is a mass diffusion process driven by density gradients. The data correlates reasonably well with both the Fischer model and the Battelle correlation at $0.2 \mathrm{MPa}$ pressure when hydrogen concentrations were below 4-5 mole \%. However, the data shows depletion rates below that predicted by the $0.2 \mathrm{MPa}$ linear fit of the Battelle data for data extrapolated to 10 mole $\%{ }^{1}$ The steady-state pressure conditions with the 1/4 scale PAR and the 1/2 scale PAR were $\sim 0.3 \mathrm{MPa}$. This means that the test data should really be compared to higher pressure theoretical depletion rate curves. Also, different PAR designs probably have different performance curves. Note that the Fischer model and the Battelle data are applicable to the NIS prototype PAR design which did not use the $0.5 \mathrm{~m}$ tall additional chimney used in the SNL PAR tests. 


\section{SUMMARY}

Hydrogen depletion rates using a scaled PAR were measured in the Surtsey test vessel at Sandia National Laboratories. The experiments determined the hydrogen depletion rate of a PAR in the presence of steam and also evaluated the effect of scale (number of cartridges) on the PAR performance at both low and high hydrogen concentrations.

The following conclusions can be made. The PAR started within 10 minutes in hot, steamy atmospheres when exposed to hydrogen concentrations in the range of 1 to 6 mole $\%$. The PAR appeared to generate a convective flow loop in the Surtsey vessel from the PAR outlet to the dome, down the Surtsey wall until reaching a height near the PAR inlet, and then returned to the PAR inlet. Hydrogen concentration was stratified in the lower part of the Surtsey vessel. This was proven by the hydrogen concentrations from the sample point located near the floor showing smaller decreases in time, as compared to the other sample points. The loop appeared to drive further downward below the PAR with higher hydrogen concentrations and also with larger scale PARs.

Assuming a well-mixed condition in the vessel, the hydrogen depletion rate is most likely proportional to scale. The Battelle and the Fischer correlations agree reasonably well within the range of the Surtsey PAR test depletion rate data. Parameters affecting scale proportionality include the well-mixed assumption in the methodology used to determine the depletion rate. If the PAR only consumes hydrogen in a small portion of the total vessel volume, the depletion rate calculation can overpredict consumption if the vessel is not well-mixed at all times. The depletion rate measurements then become scale-dependent since tests with larger scale and/or higher concentrations appear to deplete larger pockets of hydrogen within the total vessel volume.

\section{ACKNOWLEGMENT}

Sandia is a multiprogram laboratory operated by Sandia Corporation, a Lockheed Martin Company, for the United States Department of Energy under Contract DE-AC04-94AL85000.

\section{REFERENCES}

1. EPRI ALWR Program, "Qualification of Passive Autocatalytic Recombiners for Combustion Gas Control in ALWR Containments," (April 8, 1993).

2. Blanchat, T. K., Allen, M. D., M. Pilch, and R. T. Nichols, "Experiments to Investigate Direct Containment Heating Phenomena with Scaled Models of the Surry Nuclear Power Plant", NUREG/CR-6152, SAND93-2519, Sandia National Laboratories, Albuquerque, NM, (June 1994).

3. Sher, R., J. Li, and D.E. Leaver, "Models for Evaluating the Performance of Passive Autocatalytic Recombiners (PARs)," 1995 National Heat Transfer Conference, ANS Proceedings HTC, Vol. 8, Portland, OR, (August 5-9, 1995). 
4. Fischer, K., "Qualification of a Passive Catalytic for Hydrogen Mitigation," Nuclear Technology, Vol. 112, (October 1995). 


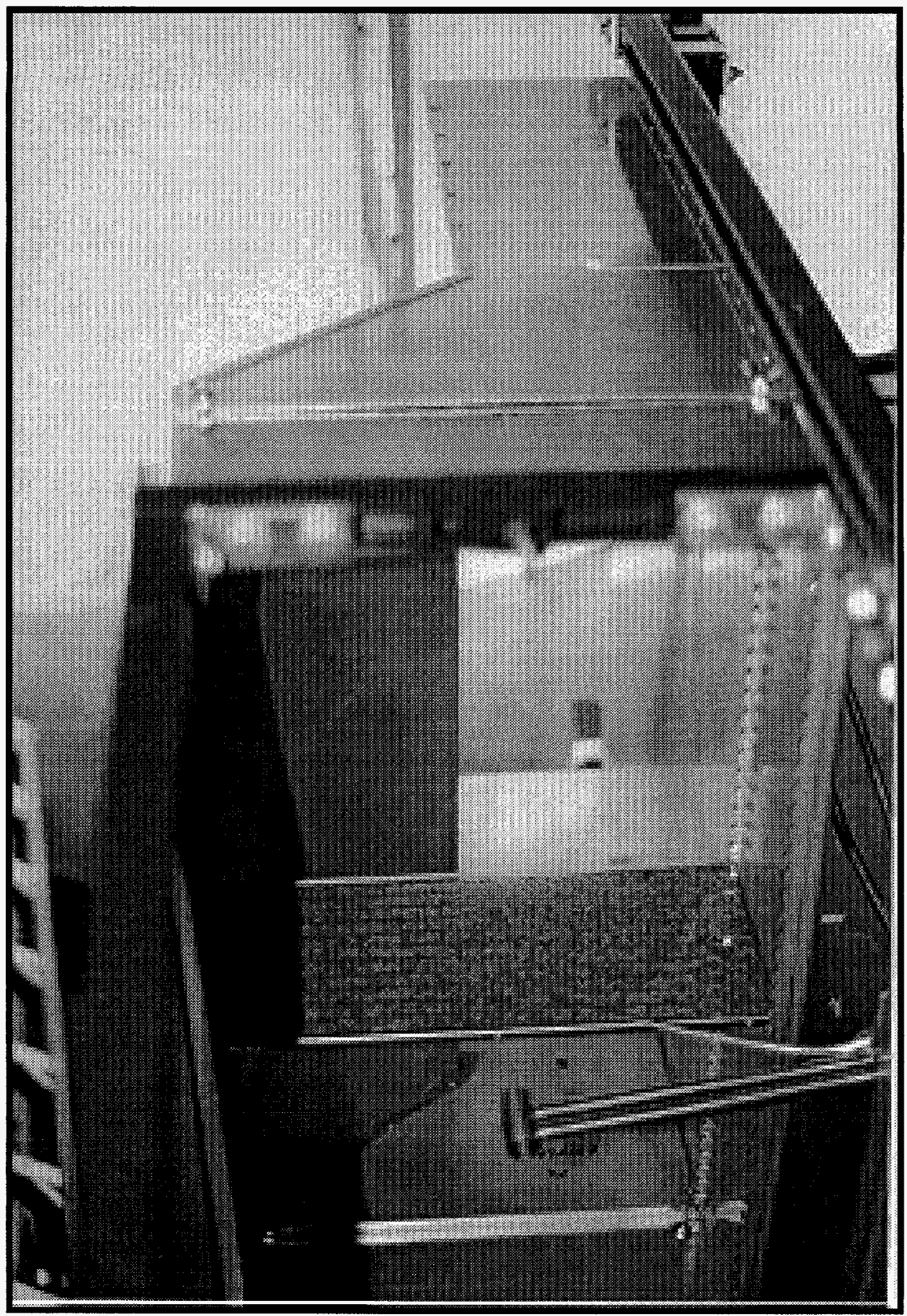

Figure 1. Bottom view of the PAR housing and chimney with one cartridge. 


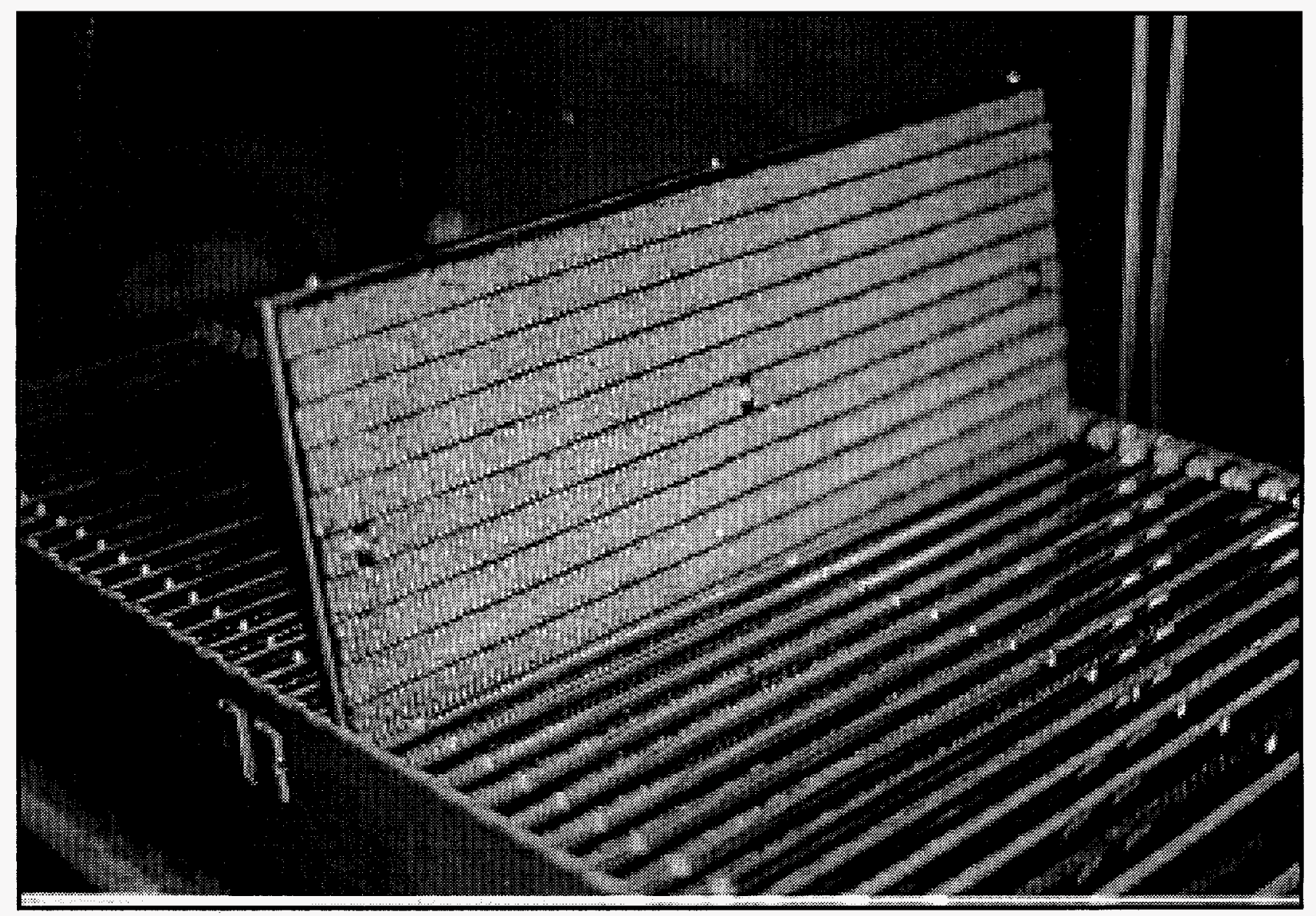

Figure 2. Cartridges held in a vertical configuration by the PAR housing. 


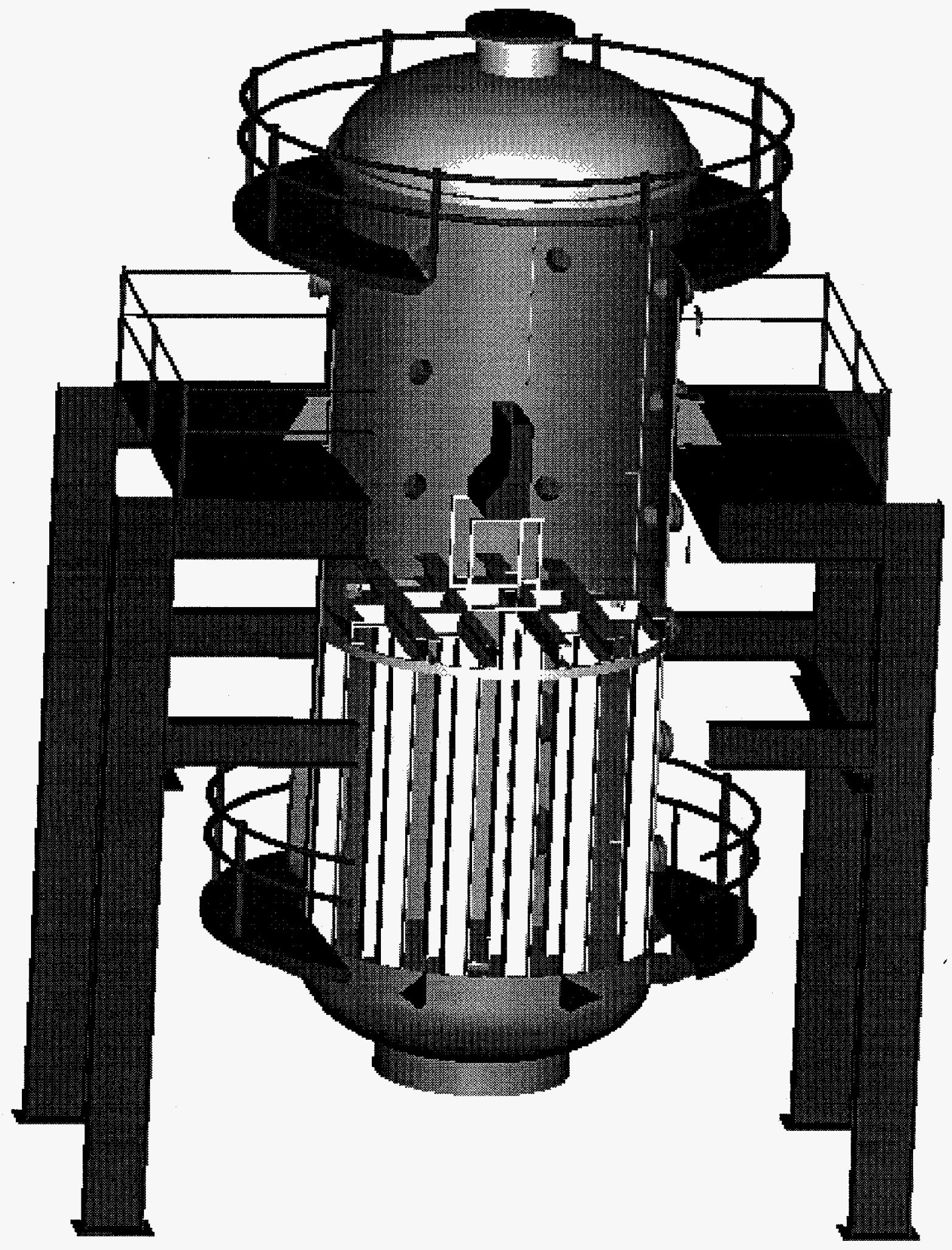

Figure 3. PAR location in the Surtsey vessel. 


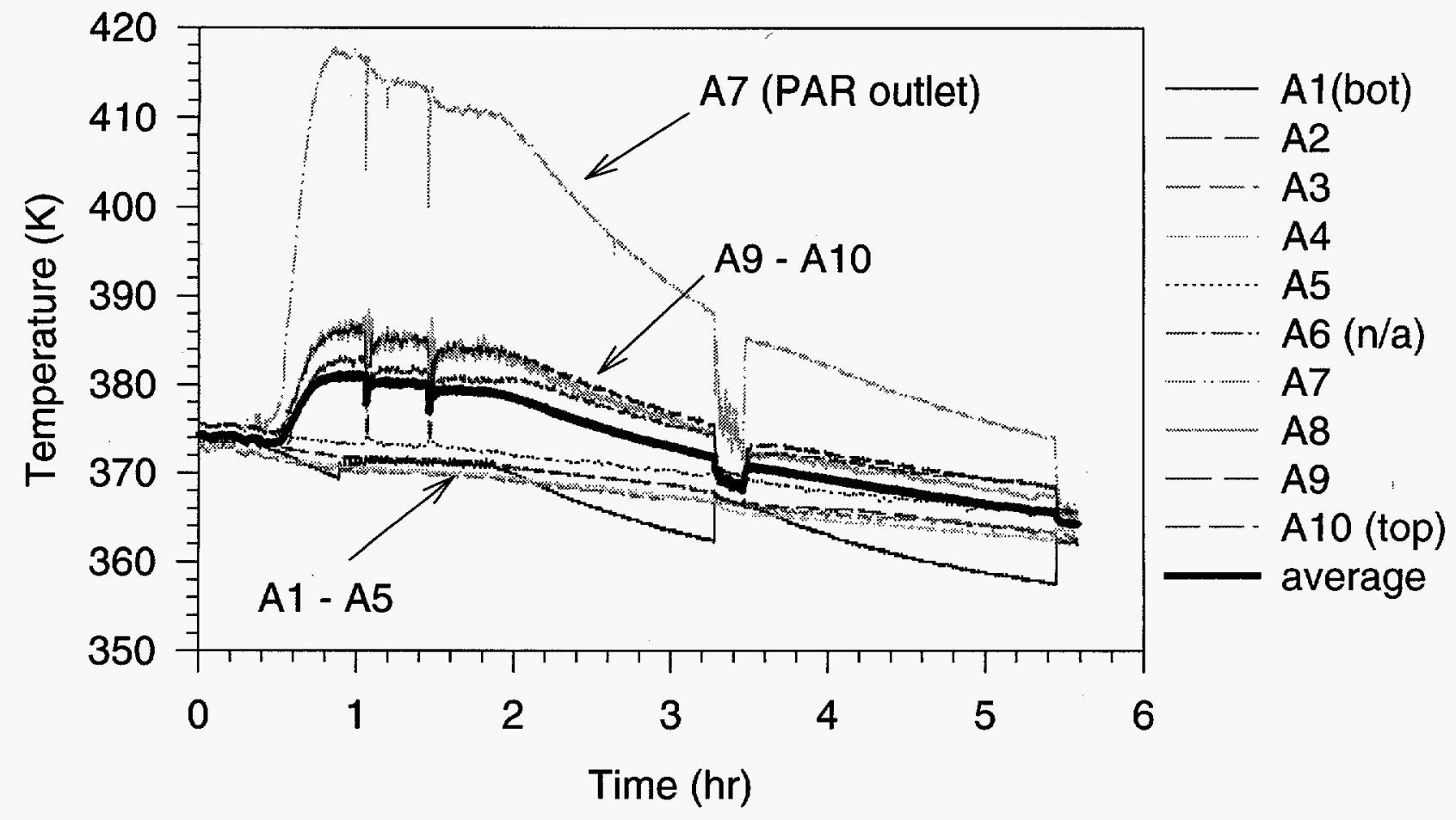

Figure 4. Centerline gas temperatures with 1/8 scale PAR. 


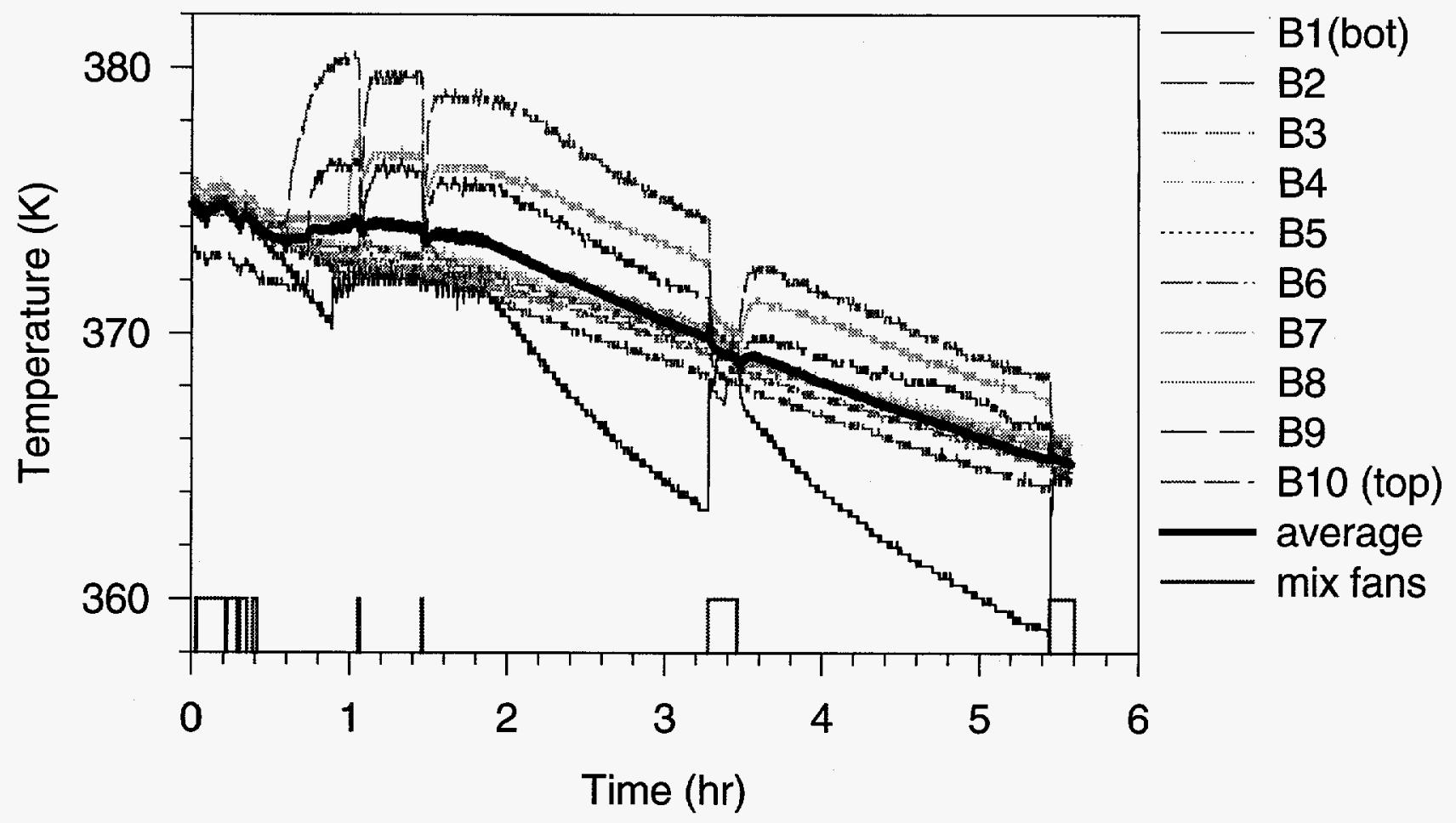

Figure 5. Wall gas temperatures with 1/8 scale PAR. 


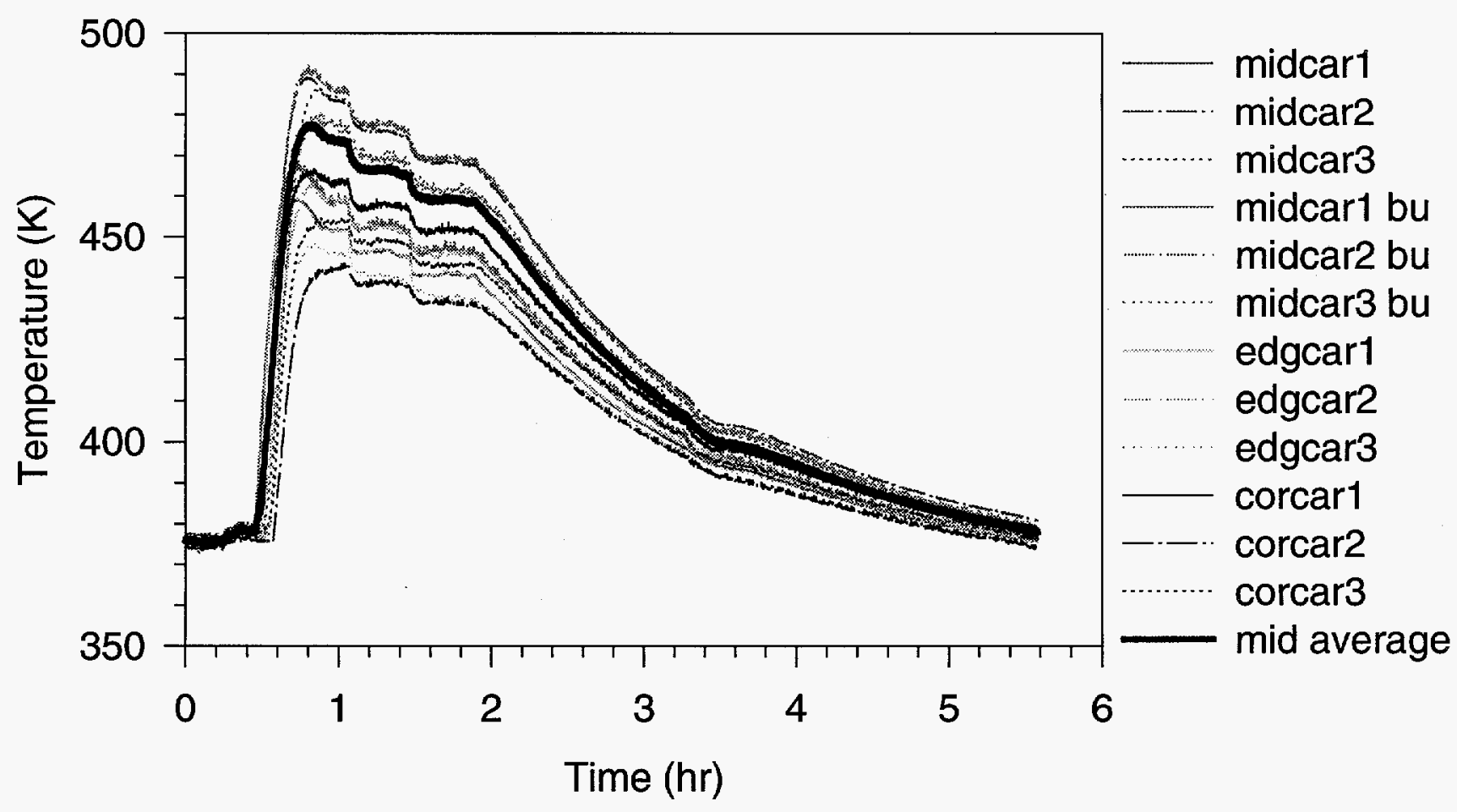

Figure 6. Catalyst cartridge temperatures with 1/8 scale PAR. 


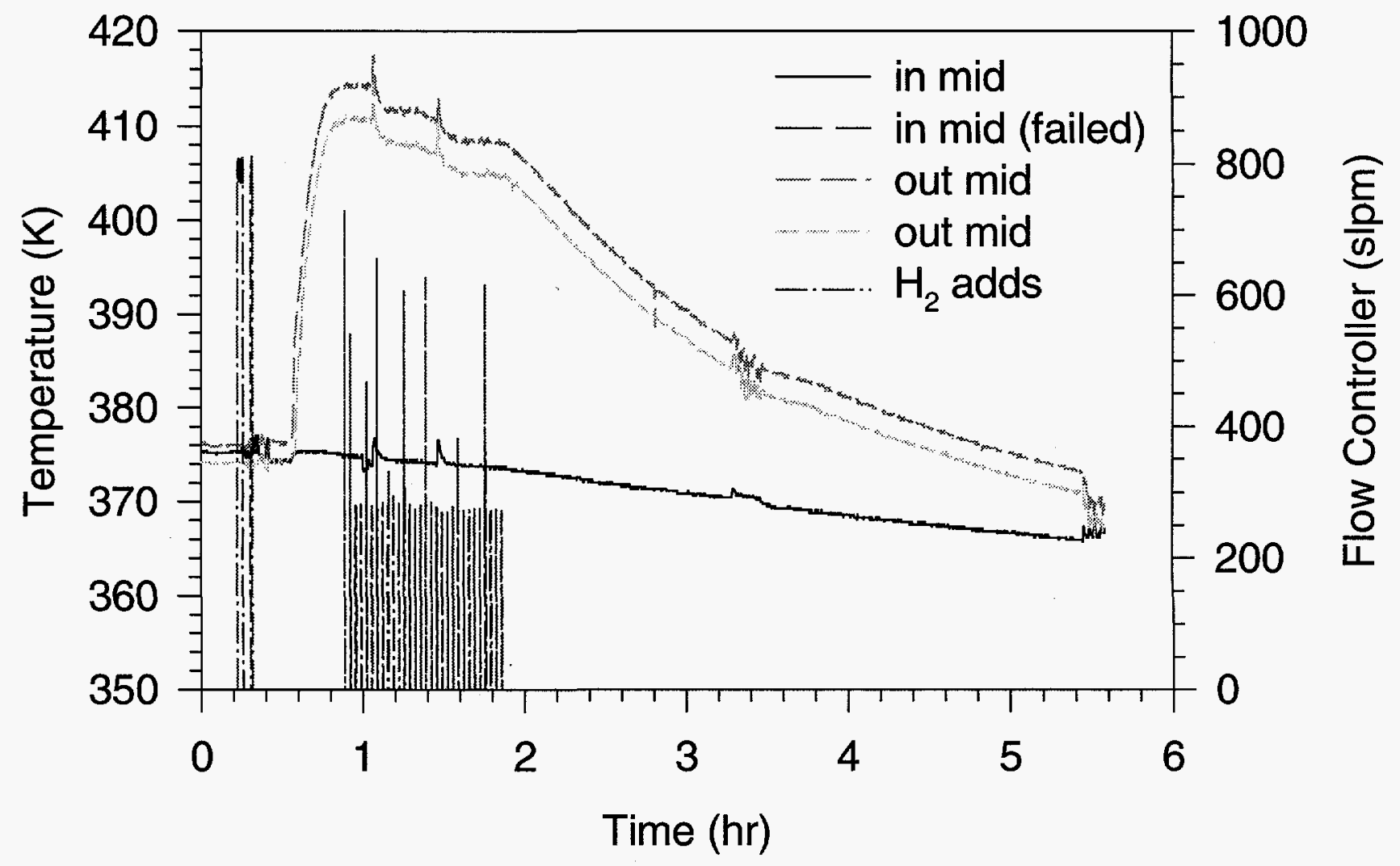

Figure 7. Inlet and outlet temperatures with $1 / 8$ scale PAR. 


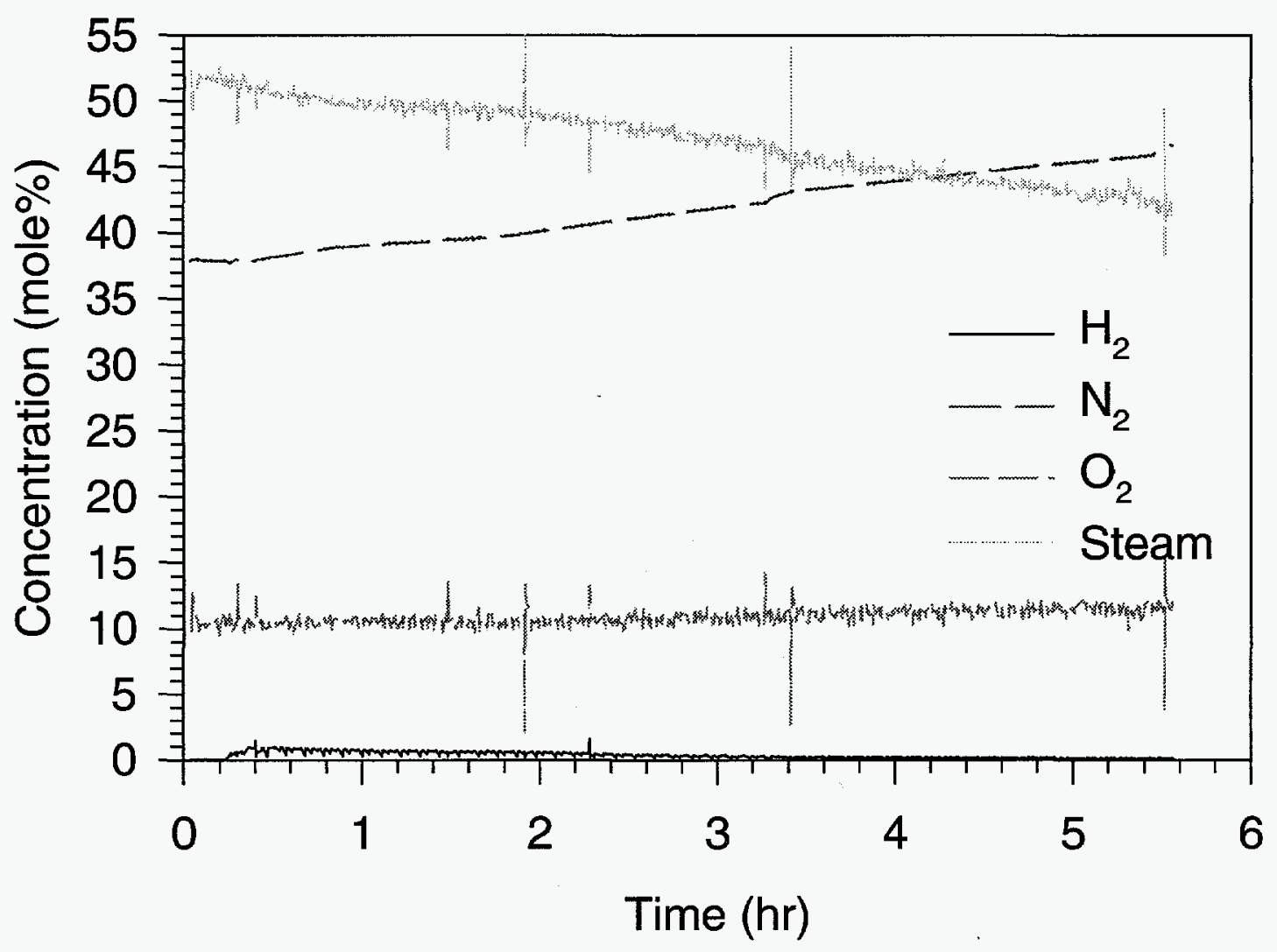

Figure 8. Gas concentrations (wet-basis) with $1 / 8$ scale PAR. 


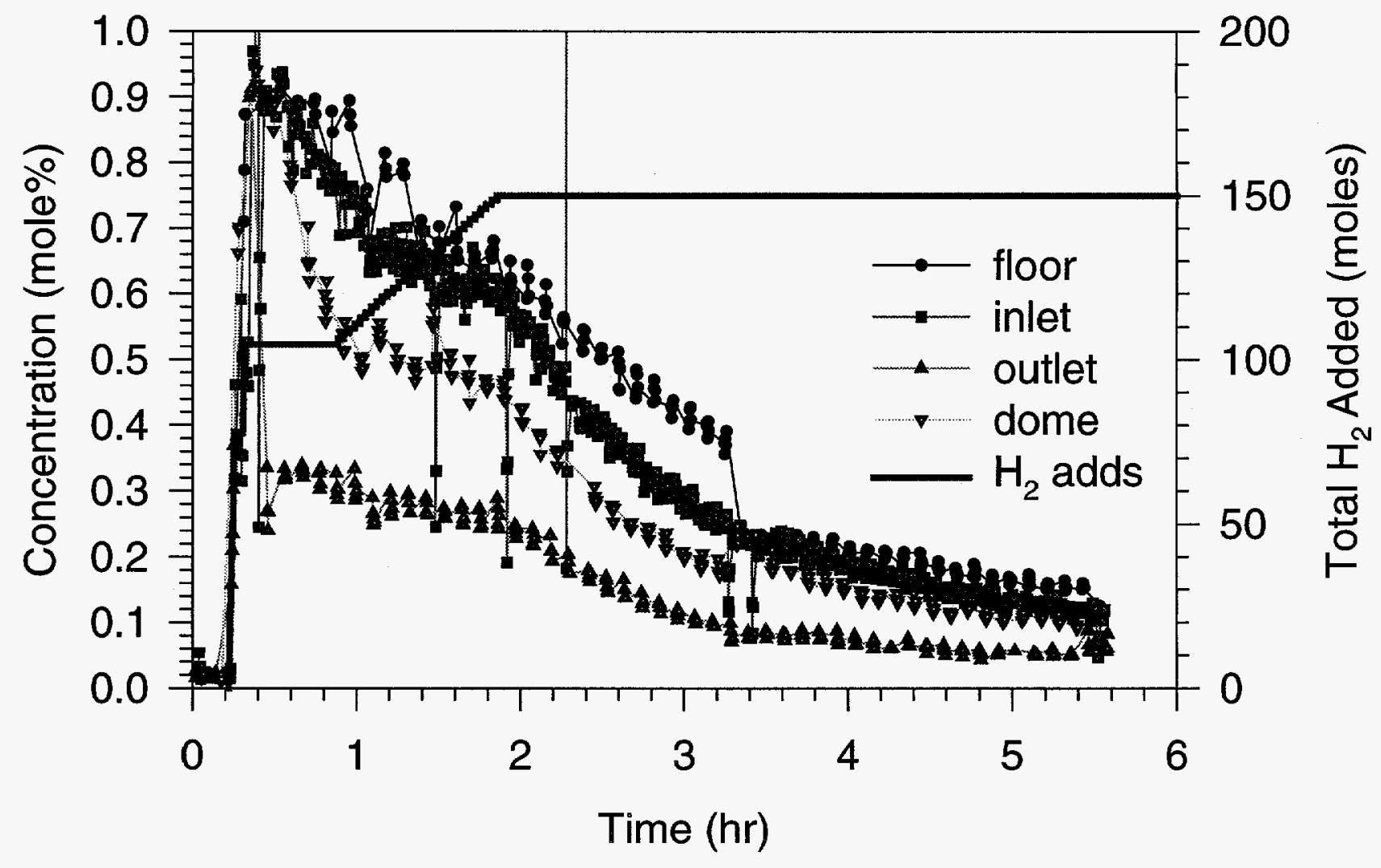

Figure 9. $\mathrm{H}_{2}$ concentrations (wet-basis) with $1 / 8$ scale PAR. 


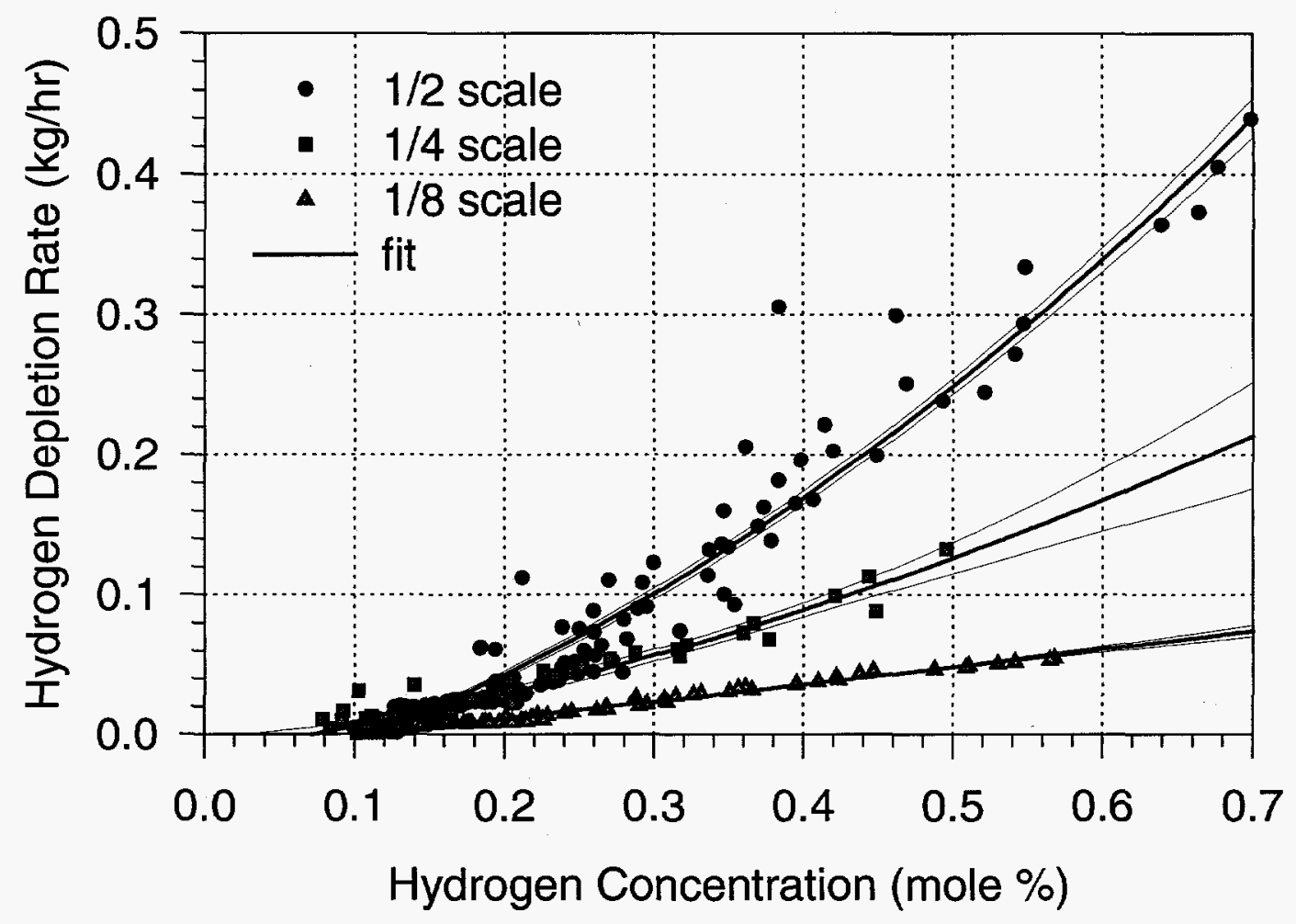

Figure 10. Hydrogen depletion rates at low hydrogen concentrations. 


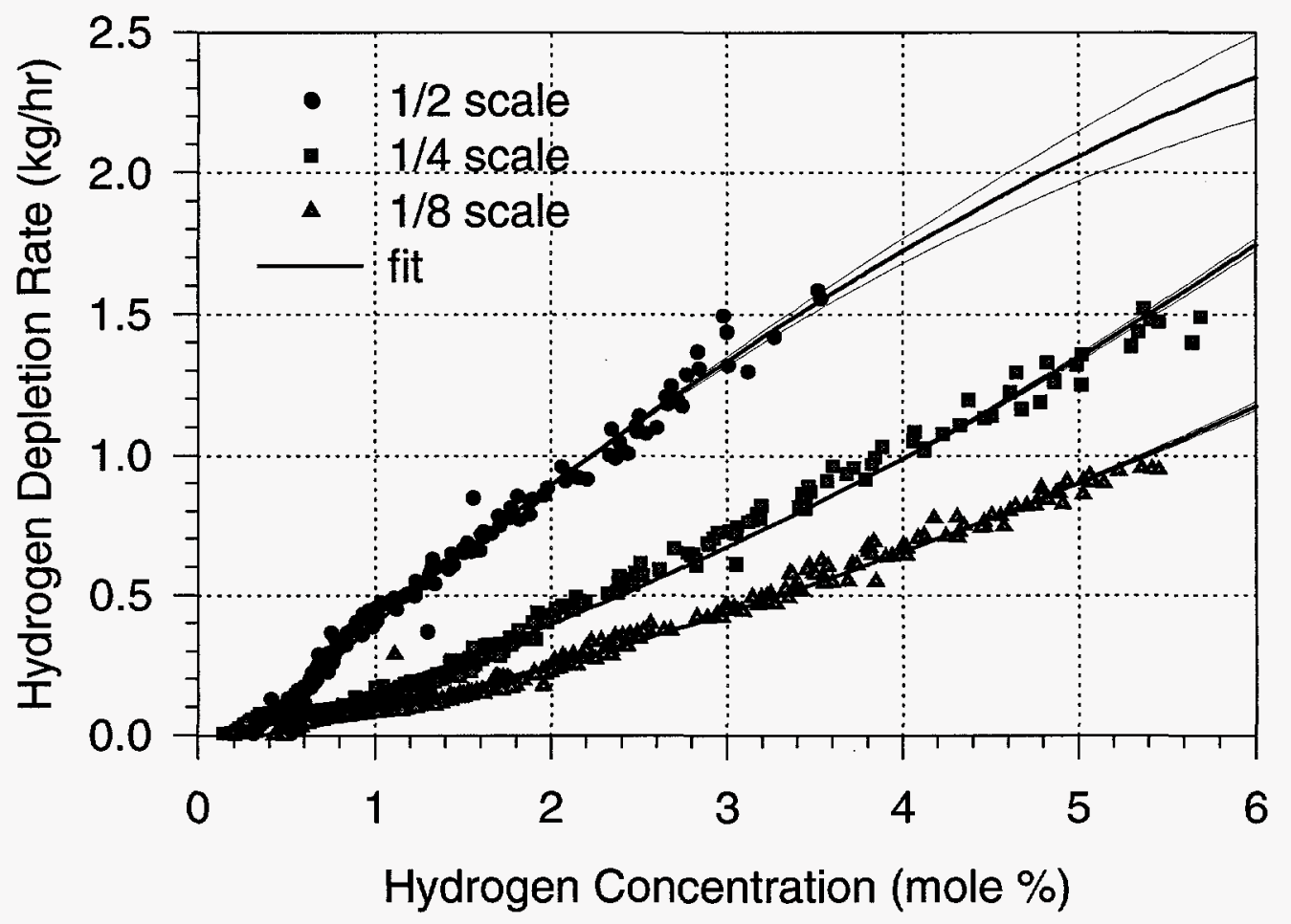

Figure 11. Hydrogen depletion rates at high hydrogen concentrations. 


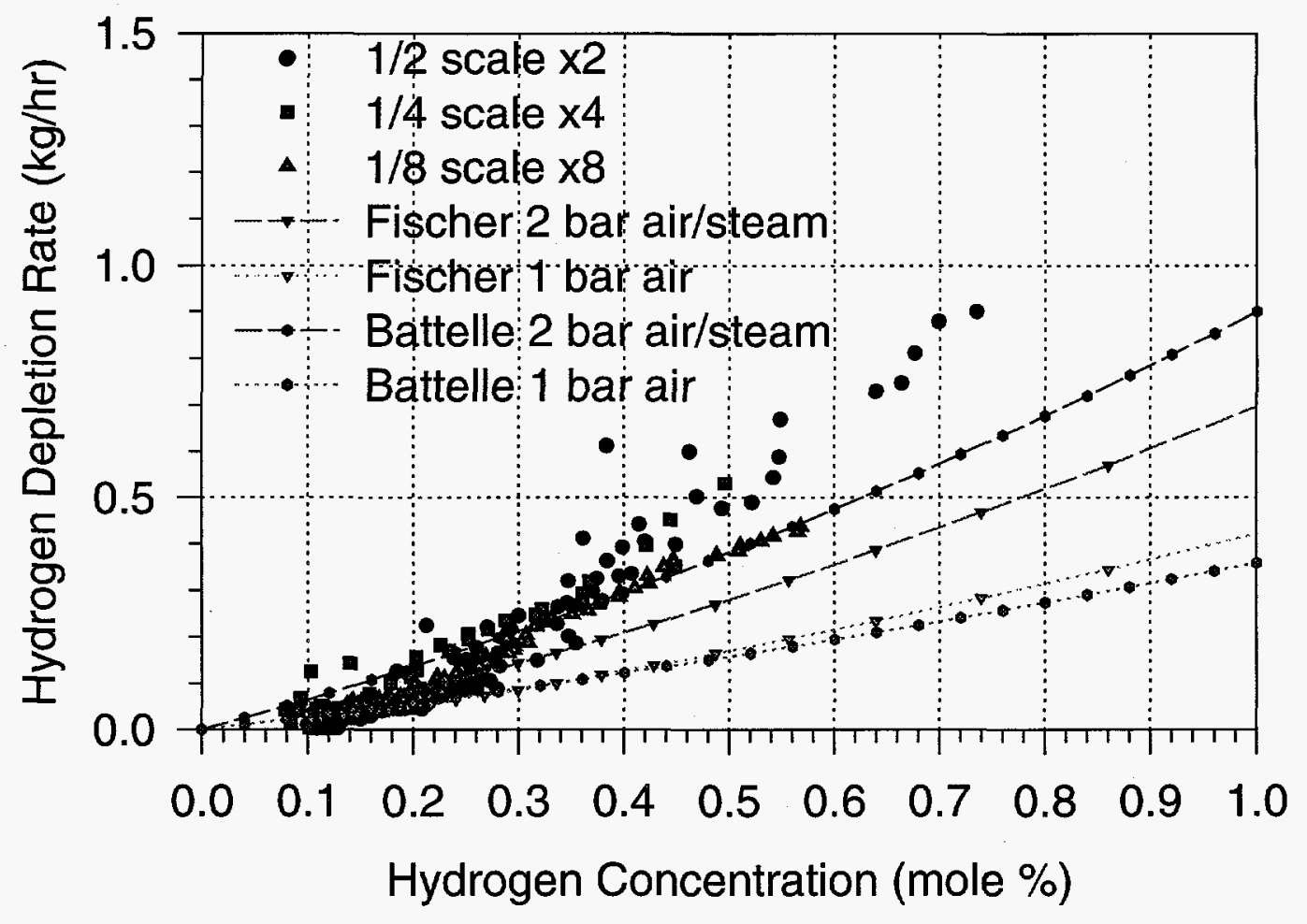

Figure 12. Normalized hydrogen depletion rates at low hydrogen concentrations. 


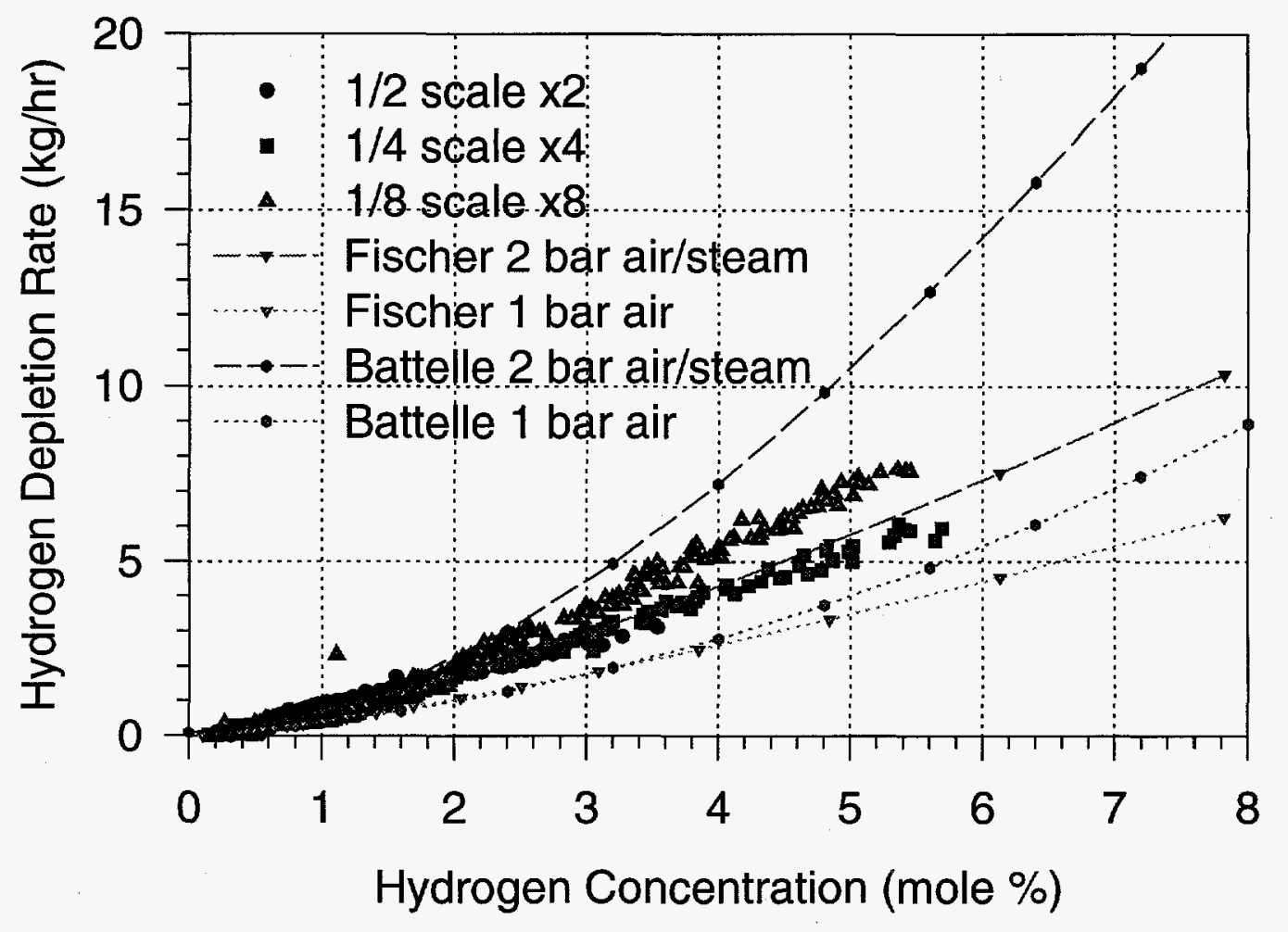

Figure 13. Normalized hydrogen depletion rates at high hydrogen concentrations. 